\title{
Particle-Based or Field-Based Models for Polymer Systems?
}

\section{Daming $\mathrm{Li}^{\star}$}

Department of Mathematics, Shanghai Jiao Tong University, Shanghai 200240, China.

Compared to the systems which are composed of simple atoms or molecules, the structures of polymer systems in equilibrium are richer. The main reason is that a polymer molecule is composed of many monomers with the same or different chemical property. Take $\mathrm{AB}$ diblock copolymers as an example. Each molecule has a form AA. - $\cdot \mathrm{ABB} \bullet \cdot \mathrm{B}$ where the monomer $\mathrm{A}$ and $\mathrm{B}$ have different chemical properties. The equilibrium structures for diblock copolymer, e.g., lamellar, hexagonal cylinder, bicontinuous gyroid and bcc sphere, etc, have been found for different composition of monomer B through computation [1] and experiments [2]. This equilibrium structures (A-rich and B-rich phase domain) with a length scale of 10-100 nm, is the result of competition of two factors: the repulsion of two chemically distinct monomer A and B, and the conformal entropy penalty. Since monomers $\mathrm{A}$ and $\mathrm{B}$ are connected in each molecule, monomer $\mathrm{A}$ and $\mathrm{B}$ are only be separated microscopically. Thus it is called microseparation.

There are two kinds of model, i.e., particle-based and field-based models, to study the microseparation of diblock copolymers. The difference between them is that the degrees of freedom in particle-based models are particle position, particle velocity, etc, which are compared to the (continuous) fields as the degrees of freedom in the field-based models. The start point of all models is how to describe the interaction between monomers. As mentioned above, at equilibrium, the competing effects of the interfacial energy (caused by the repulsion of chemically distinct monomers $\mathrm{A}$ and $\mathrm{B}$ ), which favors a larger domain size, and conformal entropy, which favors a smaller domain size, conspire to establish an optimal length scale for phase separation. Since all monomers occupied a volume, they cannot overlap geometrically. Hard sphere model are always used to approximate this kind of monomer interaction. All quantities, e.g., monomer density, pressure, etc, can be calculated if we know the partition function which is based on the interaction between monomers. The particle based models include the molecule dynamics, Monte Carlo methods, Brownian dynamics [3]. All these models always adopt a coarse-grained technique to study the equilibrium structures and its properties. This means that the details inside a monomer are not considered and the interaction between monomers become effective. From computational viewpoint, the computational demand of these particle-based models is proportional to the number of monomers, i.e., the number of degrees of freedom for the corresponding particle-based model.

In the particle-based model, the bead-spring model is always used to approximate each molecule. Another approximation, which is called continuous chain model, is used where the molecule is approximated by a continuous curve in three dimensions. Through this approximation, the corresponding partition function can be written as the (Feynman) path integral. The continuous curve (polymer) is analogous to a path of an electron in quantum mechanics. It should be noted that the structure of path integral can also be developed by Wiener, and it is called infinitedimensional functional integral in mathematical community [4]. This similarity between them has been noticed by Edwards and de Gennes in the 1960s and they used this tool of theoretical physics to the field of polymer science, including field theory techniques, scaling methods, and the renormalization group. Although each polymer molecule can be regarded as a continuous curve, the partition function is the summation of action over all these paths, but it is still a particle-based description! The field-based models use the fact that this particle-based partition function can be written as the functional integral over density and auxiliary field using Hubbard-Stratonovich transformation. Thus all quantities related to particle degree of freedom have been replaced by the (continuous) fields which are defined everywhere in the whole system. All quantities can be calculated from this partition function. Moreover, all continuum models should be derived from this fieldbased partition function. Unfortunately, the calculation of functional integral in this partition function is rather difficult, unless the other approximations or numerical computation are adopted.

If the fluctuation for the system is small, the mean field approximation is always used to calculate the partition function. This is called the mean field approximation (or saddle point approximation), i.e., the variation of functional with respect to its arguments vanishes. The corresponding model is called the self-consistent mean field (SCMF) model [5]. The physical meaning of this approximation for the polymer systems is to replace the environment of each chain (the interaction between this chain and other chains) by an auxiliary field and thus the interaction between monomer is replaced by the interaction of monomer and the auxiliary field. In SCMF model, the main computation is how to solve a modified diffusion equation to obtain the monomer density of one chain in the auxiliary field. The modified diffusion equation is rather similar to SchÖdinger equation, where the time variable in SchÖdinger equation is replaced by the arc parameter of the chain in the modified diffusion equation. One advantage of this field-based model over the particle-based models is that many discretization methods for (continuum) field models can be used; Moreover, the space and time resolution can be adjusted such that the degrees of freedom in the corresponding discretized model has less than that in the particlebased model. For example, if a simple difference scheme is adopted to solve this modified diffusion equation, its computational demand is proportional to the number of space mesh point time the "time step" along the chain. In SCMF model, each iteration of monomer density and auxiliary field needs the solving of the modified diffusion equation. The iteration stops until all quantities in SCMF model do not change.

Another field-based model is called the (classical) density functional theory (DFT) [5], which has the same mathematical theory with the (electric) density function theory for the description of electronic structure in quantum mechanics. The basic mathematical theory behind DFT is that the potential, i.e., the auxiliary field in SCMF, is also determined by the monomer density up to a constant. Under some

*Corresponding author: Daming Li, Associate Professor, Department of Mathematics, Shanghai Jiao Tong University, Shanghai 200240, China, E-mail: lidaming@sjtu.edu.cn

Received March 26, 2012; Accepted March 29, 2012; Published March 02, 2012

Citation: Li D (2012) Particle-Based or Field-Based Models for Polymer Systems? J Applied Computat Mathemat 1:e104. doi:10.4172/2168-9679.1000e104

Copyright: $\odot 2012$ Li D. This is an open-access article distributed under the terms of the Creative Commons Attribution License, which permits unrestricted use distribution, and reproduction in any medium, provided the original author and source are credited. 
approximation, the analytical expression of this potential can also be obtained though the monomer density. Thus generally DFT has more computationally efficient than SCMF model.

The SCMF theory becomes inaccurate if the fluctuation is rather large. In this case, the main contribution of functional integral is not entirely determined by the mean field solution, and field configuration far from saddle point approximation can make important contribution. There are also the field-based simulation methods, which are called theoretical Monte Carlo or complex Langevin dynamics, to directly attack the functional integral. These two field-based numerical methods correspond to particle-based methods, i.e, the usual Monte Carlo method and Brownian dynamics [5]. Compared to the usual Monte Carlo and Brownian dynamics, a special treatment in these two field-based numerical methods is needed to overcome the numerical sign problem. Another Tool, called renormalization group [6-8], is also used to analyze field theories that exhibit strong field fluctuation. This technique evolved from pioneering work in the fields of high energy and condensed matter physics (specifically the subject of critical phenomena), but now it become a standard field-theoretic tool in many disciplines. In the polymer physics, these technique are used to calculate the critical exponents or for larger-scale simulations by coupling with Monte Carlo method. The idea behind the renormalization group is to find the transformation of the free energy between the fine level to coarse level, especially the correspondence of coefficients between different levels if the free energy keeps the same structure.

Particle-based and field-based models are two types of models for studying the equilibrium structure and its properties of polymer systems. Although the field-based models have computational and theoretical advantages over particle-based models, the particle-based models are more suitable if the atomical details become important or the polymer chain length becomes short. Sometimes, these two models should be used together, e.g., in polymer-coated nanoparticles. Now, the particle-based and field-based models are also used for many polymer systems, e.g., homopolymer, diblock copolymer, polymer blends, polymer solutions, polyelectrolyte, protein-solvent system, lipid, the dumbbell polymer coupled with flow, polymers under the effect of disorder, polymer-coated nanoparticles, etc.

\section{References}

1. Matsen MW, Bates FS (1996) Unifying Weak- and Strong-Segregation Block Copolymer Theories. Macromolecules 29: 1091-1098.

2. Khandpur AK, Foerster S, Bates FS, Hamley IW, Ryan AJ et al. (1995) Polyisoprene-Polystyrene Diblock Copolymer Phase Diagram near the OrderDisorder Transition. Macromolecules 28: 8796-8806.

3. Kotelyanskii M, Theodorou DN (2004) Simulation Methods for Polymers Marcel Dekker, New York.

4. Koval'chik IM (1963) The Wiener integral. Russ Math Surv 18: 97.

5. Fredrickson GH (2006) The Equilibrium Theory of Inhomogeneous Polymers Oxford University Press, USA.

6. Wilson KG (1980) In Recent Developments of Gauge Theories. Plenum, New York.

7. Amit DJ (1984) Field Theory, the Renormalization Group and Critical Phenomena. World Scientific, Singapore.

8. Goldenfeld N (1992) Lectures on Phase Transitions and the Renormalization Group. Addison-Wesley, New York. 